University of Wollongong

Research Online

Faculty of Engineering and Information

Faculty of Engineering and Information

Sciences - Papers: Part A

Sciences

$1-1-2015$

A new approach to reduce the non-linear characteristics of a stressed power system by using the normal form technique in the control design of the excitation system

\author{
Hadi Lomei \\ University of Wollongong, hl123@uowmail.edu.au \\ Danny Sutanto \\ University of Wollongong, soetanto@uow.edu.au \\ Kashem M. Muttaqi \\ University of Wollongong, kashem@uow.edu.au \\ Mohsen Assili \\ Shahrood University of Technology
}

Follow this and additional works at: https://ro.uow.edu.au/eispapers

Part of the Engineering Commons, and the Science and Technology Studies Commons

Research Online is the open access institutional repository for the University of Wollongong. For further information contact the UOW Library: research-pubs@uow.edu.au 


\title{
A new approach to reduce the non-linear characteristics of a stressed power system by using the normal form technique in the control design of the excitation system
}

\begin{abstract}
In this paper, a new approach is presented to reduce the nonlinear characteristics of a stressed power system by reducing its second-order modal interaction through retuning some parameters of the generator excitation system. In order to determine the second-order modal interaction of the system, a new index on nonlinearity is developed using normal form theory. Using the proposed index of nonlinearity, a sensitivity function is formed to indicate the most effective excitation system parameters in the nonlinear behavior of the system. These dominant parameters are tuned to reduce the secondorder modal interaction of the system and to reduce the index on nonlinearity. The efficiency of the proposed method is validated using a four-machine two-area test system. Simulation results show that a proper tuning of the excitation controller can reduce the second-order modal interaction of the system and can even improve the transient stability margin of the network.
\end{abstract}

\section{Keywords}

reduce, non, linear, characteristics, stressed, power, system, normal, approach, form, excitation, technique, control, design

Disciplines

Engineering | Science and Technology Studies

\section{Publication Details}

H. Lomei, D. Sutanto, K. M. Muttaqi \& M. Assili, "A new approach to reduce the non-linear characteristics of a stressed power system by using the normal form technique in the control design of the excitation system," in Industry Applications Society Annual Meeting, 2015 IEEE, 2015, pp. 1-6. 


\section{A New Approach to Reduce the Non-Linear Characteristics of a Stressed Power System by Using the Normal Form Technique in the Control Design of the Excitation System}

\author{
Hadi Lomei*, Danny Sutanto and \\ Kashem M. Muttaqi \\ School of Electrical, Computer and \\ Telecommunications Engineering \\ University of Wollongong, NSW, Australia \\ "hl123@uow.edu.au
}

\author{
Mohsen Assili \\ Department of Electrical and Robotic Engineering \\ Shahrood University of Technology, Iran \\ m.assili@shahroodut.ac.ir
}

\begin{abstract}
In this paper, a new approach is presented to reduce the nonlinear characteristics of a stressed power system by reducing its second-order modal interaction through retuning some parameters of the generator excitation system. In order to determine the second-order modal interaction of the system, a new index on nonlinearity is developed using normal form theory. Using the proposed index of nonlinearity, a sensitivity function is formed to indicate the most effective excitation system parameters in the nonlinear behavior of the system. These dominant parameters are tuned to reduce the second-order modal interaction of the system and to reduce the index on nonlinearity. The efficiency of the proposed method is validated using a four-machine two-area test system. Simulation results show that a proper tuning of the excitation controller can reduce the second-order modal interaction of the system and can even improve the transient stability margin of the network.
\end{abstract}

Index Terms -index of nonlinearity; modal interaction; nonlinear systems; normal form; transient stability.

\section{INTRODUCTION}

Today Power systems are increasingly stressed due to the continuously growing load demand and less investment in system expansion. In such a stressed condition, conventional local linear model tested only at equilibrium points may fail to model the complete system characteristics or may reveal incomplete system performance. As the system become more stressed, complicated phenomena involving the interaction between the system modes may occur [1]. As discussed in [2], the interaction between modes plays an important role in the dynamic characteristics of the system and must be considered in comprehensive dynamic studies.

In the late eighties, attempts to consider the nonlinear characteristics and modal interaction of the system were initiated. In [1] and [3], the Envelope Equation, which governs the aspect of the resonance between two modes, is presented. In [2], the inter-area mode phenomenon in stressed power systems following large disturbances is studied. The importance of adding second order terms using a time domain simulation is presented in [4]. In the same year, Normal Form (NF) theory is used as a tool to identify the nonlinear aspects of the modal interaction phenomenon [5].

Over the past few years, NF has been widely used to determine the effect of the modal interaction on controller's performance of the system [6] - [8]. In [9] and [10], some guidelines were presented to design system controllers and reduce the inter-area oscillation of the system using NF. NF is also used to indicate the effect of modal interactions on machine states, system participation factors and eigenvectors [11]-[12], [13]. In a stressed power system, NF theory is used to study the nonlinear modal interaction [14] and its influence on system controller design and allocation [15]-[17]. Most recently, the effect of the modal interaction with higher than 2nd order in small-signal analysis is investigated [18]. In [19] and [20], an attempt is made to derive the closed-form nonlinear solutions of torques and their modal interactions to nonlinear torsional dynamics.

This paper investigates a new method to reduce the secondorder modal interaction of a power system by retuning the excitation system parameters. To determine the nonlinear characteristics of the system, a new index of nonlinearity is developed using the NF technique. The index is then used to determine the most effective excitation system parameters, in addition to the direction of their influence in the second-order modal interaction using a sensitivity function. Then these parameters are retuned in regards to their impact on the index of nonlinearity. The new parameters are shown to be successful in reducing the second-order modal interaction through a time domain simulation. The simulation results also show that the reduction in the second-order model interaction will increase the transient stability boundary of the network and improve its power transfer and generating capability under a stressed operating condition.

The paper is organized as follows. Section II describes the second order solution of the system using NF theory. Section III presents the proposed method to reduce the second-order modal interaction using a sensitivity function. Section IV reports on further investigation and results, when the proposed method is applied on a four-machine two-area test system.

\section{SECOND-ORDER SOLUTION USING NORMAL FORM}

Consider the following general nonlinear ordinary differential equation,

$$
\dot{x}=f(x)
$$

where $x$ is the vector of system states $\left[\begin{array}{llll}x_{1} & x_{2} & \ldots & x_{N}\end{array}\right]^{T}$ and $f$ is the real valued vector field.

Let the system represented by (1) be locally approximated by the Taylor series expansion at the stable equilibrium point 
$x_{S E P}$ as given in (2),

$$
\dot{x}=f(x)=\sum_{n=1}^{\infty} \frac{1}{n !}\left[\sum_{i=1}^{N} x_{i} \frac{\partial}{\partial x_{i}}\right]^{n} f\left(x_{S E P}\right)
$$

Where, $x_{i}$ is the $i^{\text {th }}$ state variable and represents the deviation from the equilibrium point.

Each term of the Taylor series expansion, at the equilibrium point of the system, indicates a specific characteristic of the nonlinear system. Using more terms in the series provides more precise and detailed modeling of the nonlinear system.

The abbreviated version of the Taylor series expansion is given by,

$$
\dot{x}=A x+F_{2}(x)+F_{3}(x)+\cdots
$$

where $F_{2}(x), F_{3}(x)$ and so on are the second, third and higher order terms of the Taylor series expansion.

Considering the first and the second order of the Taylor series, (3) can be expressed as (4):

$$
\dot{x}_{i}=A_{i} x+\frac{1}{2} X^{T} H^{i} X
$$

where $A_{i}$ is the $i^{t h}$ row of Jacobian, $A$, and $H_{i}$ is the Hessian matrix in the equilibrium point of $x_{S E P}$.

Considering the matrix $A$ as diagonalizable, and using $U$, the matrix of the right eigenvalues of $A$, (4) can be transformed to a Jordan-form frame using the similarity transformation $X=U Y$ as expressed in (5),

$$
\dot{y}_{j}=\lambda_{j} y_{j}+\sum_{k=1}^{N} \sum_{l=1}^{N} C_{k l}^{j} y_{k} y_{l}
$$

where,

$$
C^{j}=\frac{1}{2} \sum_{k=1}^{N} V_{j l}^{T}\left[U^{T} H^{l} U\right]=\left[C_{k l}^{j}\right]
$$

and $V$ is the matrix of the associated left eigenvectors. If the non-resonance condition, $\lambda_{i} \neq \lambda_{k}+\lambda_{l}$, holds for all eigenvalues of $A$, then the normal form transformation of (5) is defined by,

$$
y=z+h_{2}(z)
$$

where, $h_{2}$ contains only polynomial terms.

The elements of the second order transformation for a power network are given in [9] as expressed in (8),

$$
h_{2}^{j}(Z)=\sum_{k=1}^{N} \sum_{l=1}^{N} h_{2 k l}^{j} z_{k} z_{l}
$$

where $h_{2 k l}^{j}$ is the corresponding element of $h_{2}$ in (7) and defined by,

$$
h_{2 k l}^{j}=\frac{C_{k l}^{j}}{\lambda_{k}+\lambda_{l}-\lambda_{j}}
$$

Here, if no resonance of the second-order occurs, all second-order terms may be eliminated using the second-order transformation. In other words, a system consisting of second and first-order terms is equivalent to a purely linear system, the $\mathrm{z}$-coordination system (including the terms up to the second-order) [9]. In this coordination system, the secondorder system takes on the form presented in (10),

$$
\dot{z}_{j}=\lambda_{j} z_{j}
$$

where, $z_{j}$ is an element of the NF variable vector.

Now, the second-order NF system has been divided into two parts; its dynamics, which are presented by a set of linear decoupled equations, and a second-order polynomial variable transformation. The solution to the NF differential equation is

$$
z_{j}(t)=z_{j 0} e^{\lambda_{j} t}
$$

where, $z_{j 0}$ is the initial condition of the NF variable $z_{j}$.

This solution can be transformed back to the Jordan form as expressed in (12),

$$
y_{j}(t)=z_{j 0} e^{\lambda_{i} t}+\sum_{k=1}^{N} \sum_{l=1}^{N} h_{2 k l}^{j} z_{k 0} z_{l 0} e^{\left(\lambda_{k}+\lambda_{l}\right) t}
$$

In (12), the first term represents the individual modes of the system, while the later one shows the system second-order modal interaction. The ratio of the second term magnitude to the first term can be used to express the impact of the second order modal interaction of the system as expressed in (13),

$$
I_{j}=\frac{\left|\sum_{k=1}^{N} \sum_{l=1}^{N} h_{2 k l}^{j} z_{k 0} z_{l 0}\right|}{\left|z_{j 0}\right|}
$$

Hereafter, $I_{j}$ is called the index of nonlinearity. This index approximates the nonlinear characteristics, besides the second order modal interaction of the system.

As can be seen from (13) and (9), the index can be excited by three main factors as presented below:

1. Initial conditions (the size of $z_{k 0} z_{l 0}$ )

2. A large value of $C_{k l}^{j}$ that will cause large value in $h^{j}{ }_{2 k l}$

3 . Eigenvalues of the system $\left(\lambda_{k}+\lambda_{i} \approx \lambda_{j}\right)$

The third factor (the location of eigenvalues in the s-plane) has a tremendous impact in the second order modal interaction excitation.

\section{THE PROPOSED METHOD}

The significant influence of the system eigenvalues in the value of the index of nonlinearity is determined by (13), in which the value of the index of nonlinearity is proportional to the system eigenvalues. However, the location of the system eigenvalues in the s-plane is also highly influenced by the excitation system. So, the excitation system parameters play an important role in the nonlinear characteristics of the system. Fig. 1 shows the model of a classic excitation system. In this model, $T_{R}$ is the voltage regulator time constant, $T_{C}$ and $T_{B}$ are the compensator time constants, $K_{A}$ and $T_{A}$ are the amplifier gain and time constant respectively. $V_{P S S}$ is the auxiliary signal from power system stabiliser (PSS) and $V_{O E L}$ is the protective signal from the overexcitation limiter.

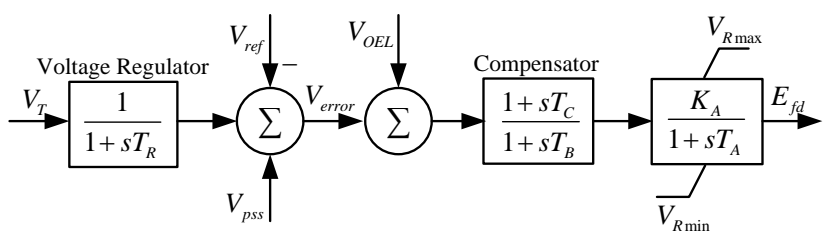

Fig. 1. The classic excitation system model block diagram. 
In the proposed method, the main accessible parameters of the excitation system, $K_{A}, T_{B}$ and $T_{C}$ are retuned to influence the nonlinear characteristics of the system in a way, which leads to the reduction of second-order modal interaction. To set the excitation model parameter efficiently, a sensitivity functions is defined by,

$$
\begin{gathered}
S_{K_{A}}=\Delta I_{j} / \Delta K_{A} \\
S_{T_{B}}=\Delta I_{j} / \Delta T_{B} \\
S_{T_{C}}=\Delta I_{j} / \Delta T_{C}
\end{gathered}
$$

where, $S$ is the sensitivity value and the $\Delta I_{j}$ is the change in the index of nonlinearity.

Due to the dependency of the dynamic studies to the operating condition of the system, a set of the most probable operating scenarios must be considered. Fig. 2 shows the algorithm of the proposed method.

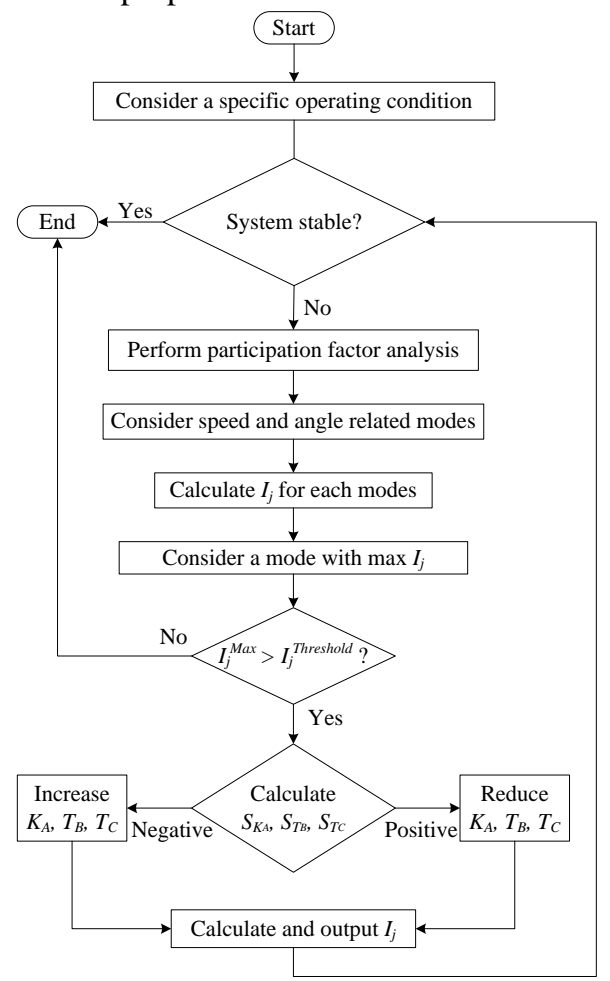

Fig. 2. Flowchart of the proposed method.

In the first step, the rotor angle stability studies are performed, and if the system is stable, the next scenario will be implemented. In the case of instability, the participation factor analysis is performed and the speed and the rotor angle related modes are identified. The proposed index of nonlinearity is then calculated for these modes and the mode with the maximum index of nonlinearity is considered as $I_{j}^{\max }$. In this step, a threshold value $I_{j}^{\text {Threshold }}$ is set. If $I_{j}^{\text {max }}<I_{j}^{\text {Threshold }}$, this mode will be neglected because of its linear behavior; otherwise, it will be considered to be highly nonlinear. To reduce the nonlinear characteristics of these modes, the exciter system parameters are redesigned. A proper exciter system design is achieved by calculating the sensitivity of the index of nonlinearity to each parameter as expressed in (14), (15) and (16). If the sensitivity of a parameter is positive, the value of that parameter is decreased and, if it is negative, the value of the parameter is increased.

\section{CASE STUDY}

The developed method is applied to a two-area, four generator test system taken from [21], which is modified to consider different operating conditions according to [17]. These two areas are connected through two parallel transmission lines. Each transmission line transfers $400 \mathrm{MW}$ of active power. Fig. 3 shows a single-line diagram of the test system. For this study, all generators are modeled by the two axis model and equipped with an exciter [22], and the nominal apparent power for each generator is assumed to be $900 \mathrm{MVA}$. The output operating voltage of each generator is $20 \mathrm{kV}$. The loads are modeled as constant impedances with no dynamics; moreover, the generators are equipped with the excitation system. As a disturbance, the generation outage at GEN4 is considered for 300 milliseconds.

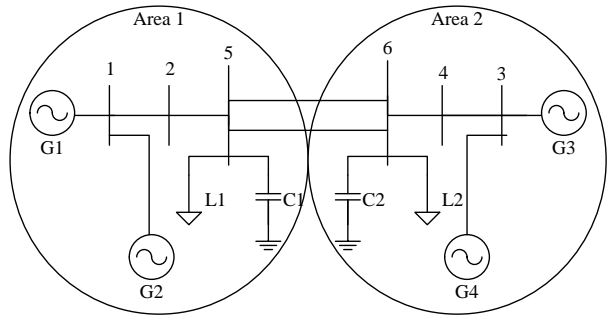

Fig. 3. Single line diagram of the test system.

First, the linear and the nonlinear characteristics of the test system are investigated using a time domain simulation and then the second-order modal interaction of the system is reduced using the new parameters for the excitation system model.

\section{A. Linear and Nonlinear Characteristics of the System}

The behavior of the linear and the nonlinear model of the test system, after the disturbance, is presented in Fig. 4.
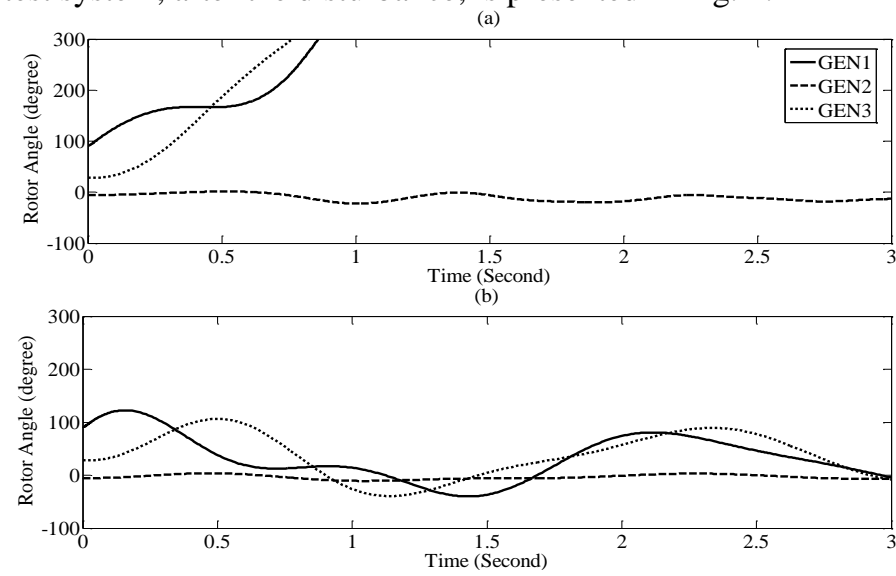

Fig. 4. Rotor angle of the generators after the disturbance, (a) nonlinear system, (b) linear system.

In Fig. 4, after the outage clearance, the rotor angles of the GEN1 and GEN3 rise significantly when using the nonlinear system model, but when using the linear system model, they are able to follow a stable route towards their initial value. This significant difference between the nonlinear and linear 
modeling of the system arises from the influence of the second-order modal interaction on the nonlinear modeling.

To determine the modes which are responsible for the second-order modal interaction in the nonlinear system, participation factor analysis is used. The participation factor analysis indicates the highest participation modes in both the speed and the rotor angle of generators. Table I represents the results of the participation factor analysis for the 3 modes with the highest values of participation factors.

TABLE I

THE PARTICIPATION OF EACH GENERATOR IN ELECTROMECHANICALLY MODES OF THE TEST SYSTEM

\begin{tabular}{|c|c|c|c|c|}
\hline Mode \# & Eigenvalue & GEN1 & GEN2 & GEN3 \\
\hline 1 & $-0.68 \pm \mathrm{j} 6.68$ & 0.25 & 0.60 & 0.47 \\
\hline 2 & $-0.67 \pm \mathrm{j} 6.65$ & 0.59 & 0.35 & 0.14 \\
\hline 3 & $-0.10 \pm \mathrm{j} 3.24$ & 0.27 & 0.033 & 0.49 \\
\hline
\end{tabular}

The results from Table I show that for the third mode, GEN1 and GEN2 have the highest participation factor while the participation factor of GEN2 is very small; so, mode 3 is originally generated from the interaction between GEN1 and GEN3. To verify this, the proposed index of nonlinearity is also used to indicate the nonlinear characteristics of the system with regards to the presented modes in Table I. Table II shows the value of the index of nonlinearity considering the mentioned electromechanical modes.

TABLE II

THE INDEX OF NONLINEARITY FOR THE SELECTED ELECTROMECHANICAL MODES

\begin{tabular}{|c|c|c|c|}
\hline Mode\# & Eigenvalue & $\left|z_{j 0}\right|$ & $I_{j}$ \\
\hline 1 & $-0.68 \pm \mathrm{j} 6.68$ & 1.70 & 0.05 \\
\hline 2 & $-0.67 \pm \mathrm{j} 6.65$ & 2.07 & 0.05 \\
\hline 3 & $-0.10 \pm \mathrm{j} 3.24$ & 2.09 & 0.72 \\
\hline
\end{tabular}

The results from Table II verify the significant role of the mode 3 in the generation of the second-order modal interaction in the system; since mode 3 has the highest value of the index on nonlinearity. In other words, the second-order modal interaction between GEN1 and GEN3 increases the nonlinear characteristics of the system.

\section{B. Reduction in the Second-order Modal Interaction}

To reduce the second-order modal interaction of the test system, the proposed approach is used to redesign the excitation system model parameters. In the first step, the sensitivity of each excitation system model parameter to the index of nonlinearity using (14), (15) and (16) is calculated. If the sensitivity of a parameter is positive, the value of that parameter is decreased, and in case of a negative sensitivity it will be increased. The magnitude of the change of a parameter is dependent on the sensitivity magnitude. The most sensitive parameter takes the most changes. Table III show the selected steps of the excitation system model parameter tuning in regards to reduce the second order modal interaction. In Table III, four sets of excitation system model parameters are provided. Table III shows the sensitivity of the parameters to the index on nonlinearity for GEN1 and GEN3 and the value of the index of the nonlinearity of the system after implementing the new parameters on the excitation system model.
TABLE III

EXCITER SYSTEM PARAMETER ALONG WITH THEIR SENSITIVITY AND INDEX OF NONLINEARITY IN FOUR EXCITATION SYSTEM MODEL PARAMETER SETTING

\begin{tabular}{|c|c|c|c|c|c|c|c|c|}
\hline \multirow{2}{*}{ Set\# } & GEN\# & $K_{A}$ & $T_{B}$ & $T_{C}$ & $S_{K_{A}}$ & $S_{T_{B}}$ & $S_{T_{C}}$ & $I_{j}$ \\
\hline \multirow{2}{*}{ Set I } & 1 & 220 & 8 & 2 & -0.0008 & 0.025 & -0.10 & \multirow{2}{*}{0.46} \\
\cline { 2 - 10 } & 3 & 210 & 9 & 1.5 & -0.0000 & 0.002 & -0.10 & \\
\hline \multirow{2}{*}{ Set II } & 1 & 240 & 7 & 3 & -0.0005 & 0.019 & -0.04 & \multirow{2}{*}{0.33} \\
\cline { 2 - 9 } & 3 & 210 & 9 & 2.5 & 0.0000 & 0.000 & -0.00 & \\
\hline \multirow{2}{*}{ Set III } & 1 & 250 & 6 & 4 & -0.0003 & 0.016 & -0.02 & \multirow{2}{*}{0.27} \\
\cline { 2 - 9 } & 3 & 210 & 9 & 3 & 0.0001 & -0.002 & 0.00 & \\
\hline \multirow{2}{*}{ Set IV } & 1 & 250 & 5.5 & 5 & - & - & - & \multirow{2}{*}{0.24} \\
\cline { 2 - 8 } & 3 & 205 & 10 & 3 & - & - & - & \multirow{2}{*}{} \\
\hline
\end{tabular}

In this test system and the considered operating scenario, GEN1 and GEN3 are responsible for the second-order modal interaction. As a result, the excitation system parameters of these two generators are considered for retuning. Figs. 5 - 8 show the rotor angles of test system generators for each set of the excitation system parameters.

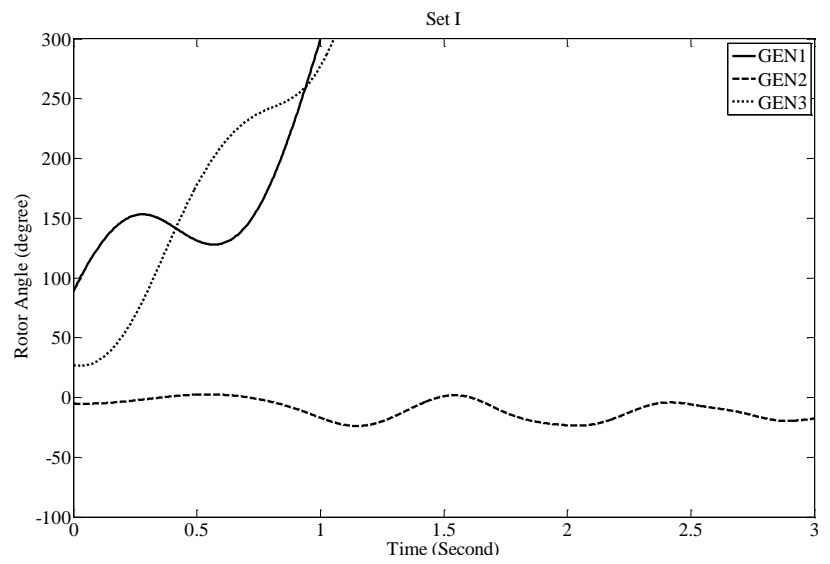

Fig. 5. Rotor angle of the generators for the exciter system parameter set I.

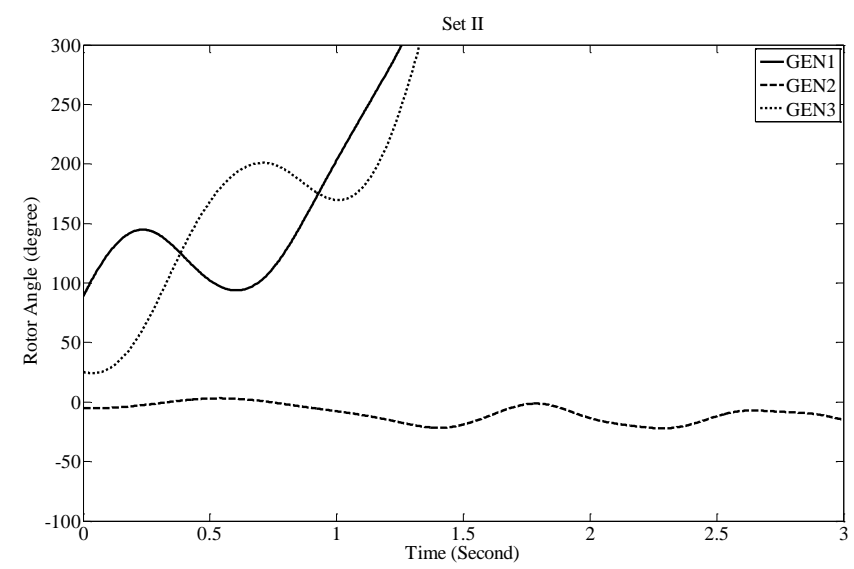

Fig. 6. Rotor angle of the generators for the exciter system parameter set II.

The time domain simulation results of the rotor angles of the test system generators presented in Figs. 5-8 show that changes in the excitation system model parameters using the proposed method significantly influence the characteristics of the system by reducing the second-order modal interaction of the system. As is evident from Figs. 5-8, as the index of nonlinearity reduces from set I to set IV, the nonlinear characteristic of the system reduces and the transient stability of the system improves. 


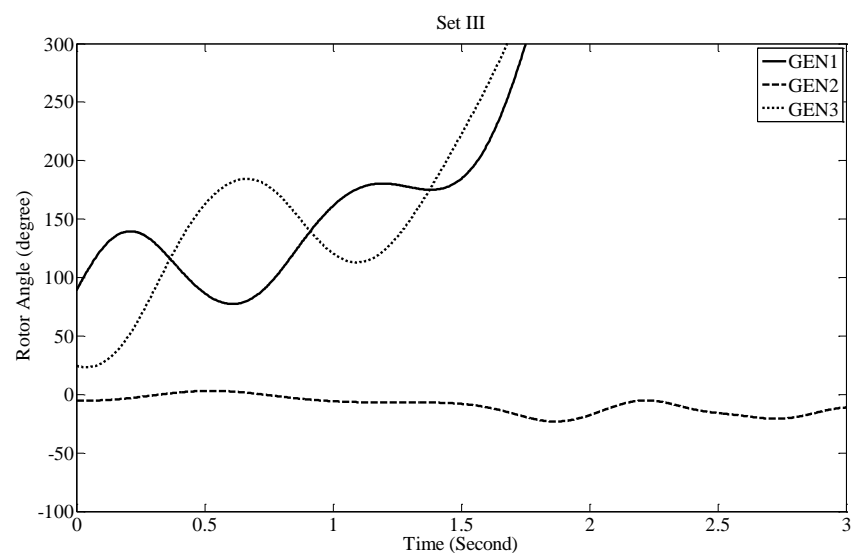

Fig. 7. Rotor angle of the generators for the exciter system parameter set III.

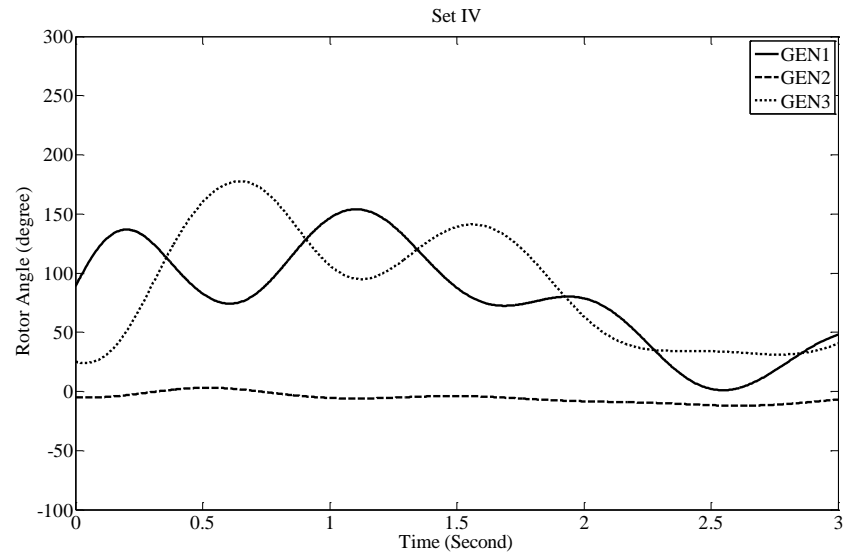

Fig. 8. Rotor angle of the generators for the exciter system parameter set IV.

\section{Expansion in the Transient Stability Boundaries}

A time domain analysis is carried out to investigate the influence of the reduction in the second-order modal interaction on the transient stability of the system. In this situation, considering the same disturbance as that of the previous section, the rotor angle behavior for four different generating operating scenarios is simulated. Table IV presents the detailed information about the four selected operating scenarios.

TABLE IV

Generator OutPut Power (MW) AT THE SELECTED OPERATING SCENARios

\begin{tabular}{|c|c|c|c|c|}
\hline Scenario & I & II & III & IV \\
\hline Case 1: conventional parameters & 560 & 602 & 607 & 700 \\
\hline Case 2: new parameters & 560 & 665 & 700 & 707 \\
\hline
\end{tabular}

The studies are carried out for two cases with different excitation system model parameters. In case 1 , the excitation system parameter of set I given in Table II is considered, in which the index of nonlinearity of the system is 0.46 . In case 2, the exciter system parameters of set IV given in Table II are considered, in which the index of nonlinearity of the system is 0.24 . Each scenario was obtained by changing the active power output of GEN1 and GEN3. In this study, the active power transfer between Area 1 and Area 2 remains constant; since the inter-area oscillation is heavily dependent on the power transfer between areas.

Case 1: In this case, the time domain simulation results for
GEN1 and GEN3 equipped with set I excitation system model parameters after the disturbance are presented in Figs. 9 - 10. The system is able to remain stable for scenarios I and II but after 5MW increase in the output active power of both GEN1 and GEN3 in scenario III, the system exceeds its transient stability boundary and becomes unstable.

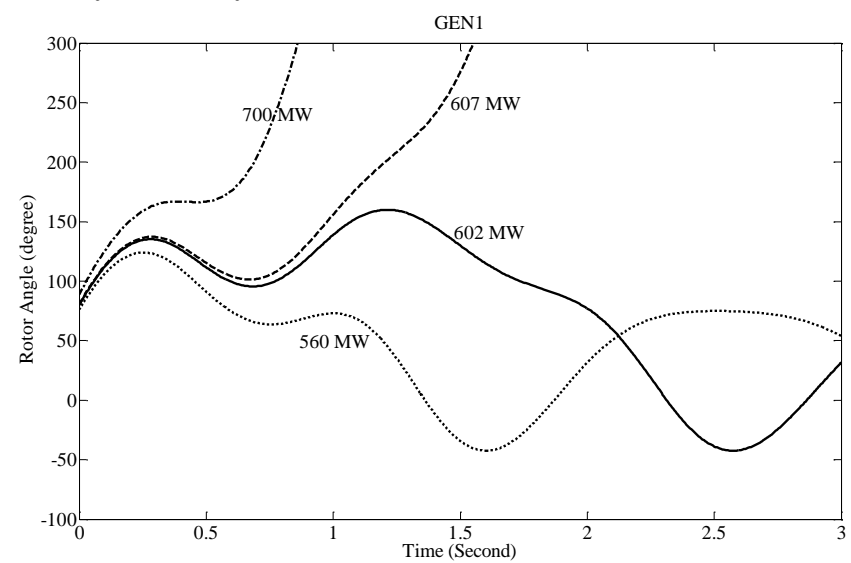

Fig. 9. Rotor angle of GEN1 after the event for different active power output scenarios with conventional exciter system parameters.

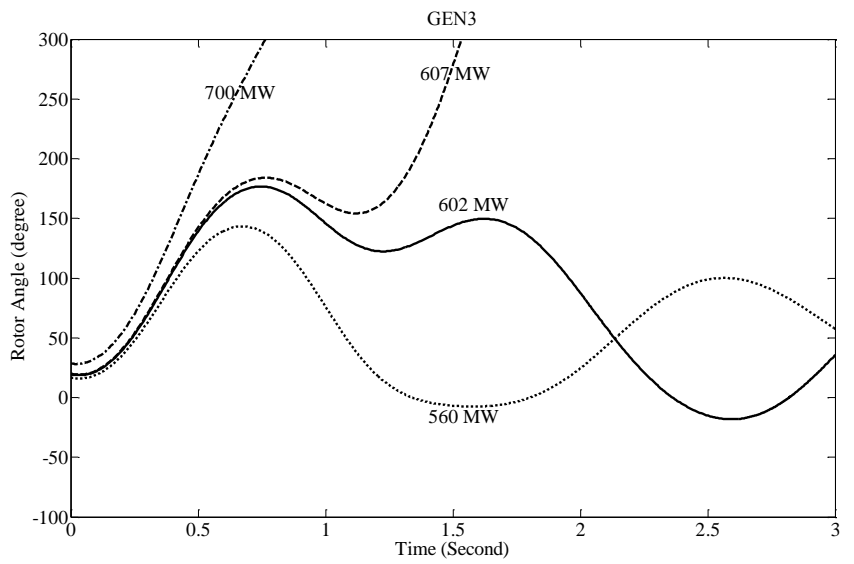

Fig. 10. Rotor angle of GEN3 after the event for different active power output scenarios with conventional exciter system parameters.

Case 2: In this case, GEN1 and GEN3 are equipped with the excitation system model parameters of set IV. These parameters are redesigned with the objective of reducing the second-order modal interaction.

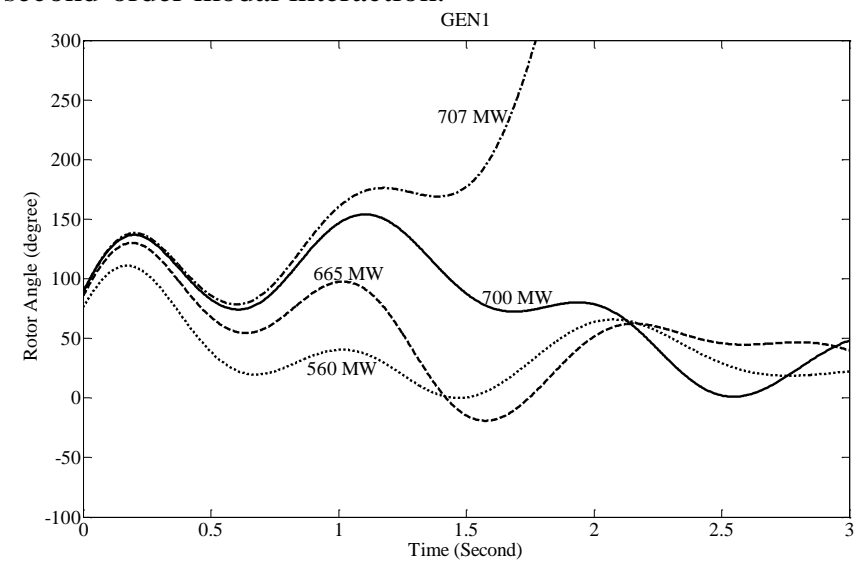

Fig. 11. Rotor angle of GEN1 after the event for different output active power scenarios with new exciter system parameters. 
Figs. 11-12 show that the transient stability boundary of the test system has been increased due to the reduction of the index of nonlinearity by using the new set of excitation system parameters. In other words, the transient stability margin of the system is improved by redesigning the exciter system parameters.

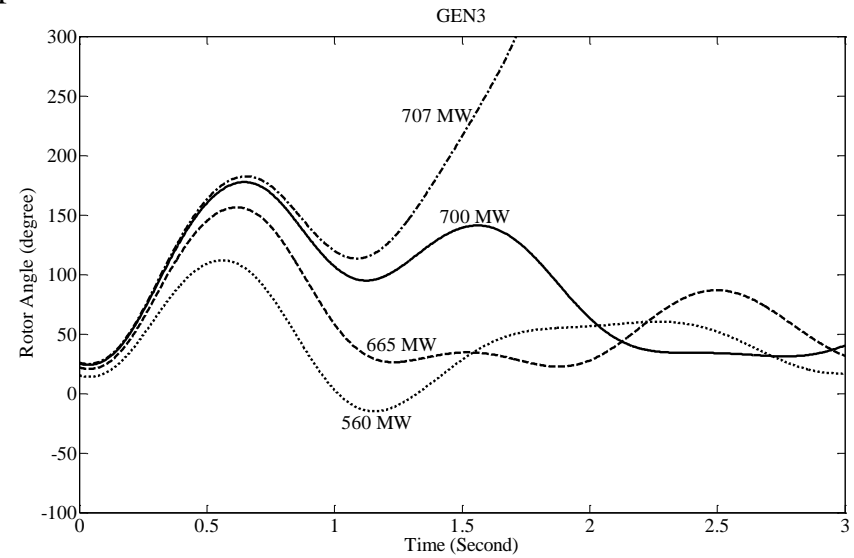

Fig. 12. Rotor angle of GEN3 after the event for different output active power scenarios with new exciter system parameters.

For this test system, the transient stability boundary of a single generator is increased by about $100 \mathrm{MW}$. In other words, by using the new set of excitation system parameters, the power generation and transfer capacity of the system may increase by about $15 \%$.

\section{CONCLUSION}

In this paper, a new method is presented to reduce the second-order modal interaction of the system. A new index of nonlinearity is developed which can indicate the nonlinear characteristics of the system due to the interaction between two pairs of modes. It is shown that by retuning the adjustable parameters of the excitation system, this index will reduce significantly and the behavior of the system move toward linear characteristics. The proposed approach has been evaluated on a four-machine, two-area test system. Detailed numerical simulation results indicate that a proper tuning of the excitation system parameters can expand the transient stability margin of the system.

\section{REFERENCES}

[1] N. Yorino, H. Sasaki, Y. Tamura, and R. Yokoyama, "A generalized analysis method of auto-parametric resonances in power systems," IEEE Trans. Power Syst., vol. 4, no. 3, pp. 1057-1064, Aug. 1989.

[2] V. Vittal, N. Bhatia, and A. A. Fouad, "Analysis of the interarea mode phenomena in power systems following large disturbances," IEEE Trans. Power Syst., vol. 6, no. 2, pp. 1515-1521, Apr. 1991.

[3] Y. Tamura and N. Yorino, "Possibility of hetero \& auto parametric resonance in power systems and their relationships with long-term stability," IEEE Trans. Power Syst., vol. 2, no. 4, pp. 890-897, Nov. 1987.

[4] S. K. Starrett, W. Kliemann, V. Vittal, and A. A. Fouad, "Power system modal behavior: significance of second and third order nonlinear terms", Proceeding of the 1993 North American Power Symposium, Washington D. C., 1993.

[5] EPRI Final Report No. TR-103704 (RP8010-28), “Analysis of stressed interconnected power networks", Mar. 1994.

[6] C. M. Lin, V. Vittal, W. Kliemann, and A. A. Fouad, "Investigation of modal interaction and its effects on control performance in stressed power systems using normal forms of vector fields," IEEE Trans. Power Syst., vol. 11, no. 2, pp. 781-787, May 1996.

[7] Y. Ni, V. Vittal, W. Kliemann, and A. A. Fouad, "Nonlinear modal interaction in HVDC/AC power systems with DC power modulation" IEEE Trans. Power Syst., vol. 11, no. 4, pp. 2011-2017, Nov. 1996.

[8] Thapar, J., V. Vittal, W. Kliemann, and A. A. Fouad, "Application of normal form of vector fields to predict interarea separation in power systems", IEEE Trans. Power Syst., vol. 12, no 2, pp. 844-850, May 1997.

[9] G. Jang, V. Vittal and W. Keliemann, "Effect of nonlinear modal interaction on control performance: use of normal form techniques in control design part I: case studies" IEEE Trans. Power Syst., vol. 13, no 2, pp. 408-413, May 1998.

[10] G. Jang, V. Vittal and W. Keliemann, "Effect of nonlinear modal interaction on control performance: use of normal form techniques in control design part I: general theory and procedure" IEEE Trans. Power Syst., vol. 13, no 2, pp. 401-407, May 1998.

[11] S. Zhu, V. Vittal, and W. Kliemann, "Analyzing dynamic performance of power systems over parameter space using normal forms of vector fields parts I: identification of vulnerable regions , IEEE Trans. Power Syst., vol. 16, no. 3, pp. 451-455, Aug. 2001.

[12] S. Zhu, V. Vittal, and W. Kliemann, "Analyzing dynamic performance of power systems over parameter space using normal forms of vector fields parts II: comparison of the system structure," IEEE Trans. Power Syst., vol. 16, no. 3, pp. 451-455, Aug. 2001.

[13] S. K. Starrett, and A. A. Fouad, "Nonlinear measures of mode-machine participatons", IEEE Trans. Power Syst., vol. 13, no 2, pp. 389-394, May 1998.

[14] E. Barocio and A. R. Messina, "Analysis of nonlinear modal interaction in stressed power systems with SVCs", Proc. IEEE Power Engineering Society Winter Meeting, vol. 2, pp. 1164 - 1169, 2002.

[15] A. R. Messina and V. Vittal, "Assessment of nonlinear interaction between nonlinearly coupled modes using higher-order spectra", IEEE Trans. Power Syst., vol. 20, no. 1, pp.375 -383, Aug. 2005.

[16] Sh. Liu, A.R. Messina and V. Vittal, "A normal form analysis approach to siting power system stabilizers (PSSs) and assessing power system nonlinear behavior", IEEE Trans. Power Syst., vol. 21, no. 4, pp. 17551762, Nov. 2006.

[17] Sh. Liu, A. R. Messina and V. Vittal, "A normal form analysis approach to siting power system stabilizers (PSSs) and assessing power system nonlinear behavior", Proc. IEEE Power Eng. Soc. General Meeting, 2006.

[18] Q. Huang, Zh. Wang and Ch. Zhang, "Evaluation of the effect of modal interaction higher than 2nd order in small-signal analysis", Proc. IEEE Power Eng. Soc. General Meeting, pp. 1-6, July 2009.

[19] W. Chen, T. Bi, Q. Yang and J. Deng, "Analysis of nonlinear torsional dynamics using second-order solutions," IEEE Trans. Power Syst., vol. 25, no. 1, pp. 423-432, Feb. 2010.

[20] T. Bi, W. Chen and Q. Yang, "Predicting non-linear torsional dynamics using second-order solutions," IET Gener. Transm. Distrib., vol. 6, iss. 4, pp. 331-338, Apr. 2012.

[21] M. Assili, "Reduction of nonlinear modal interaction and its effect on stability of power system", M.Sc. dissertation, Dept. Elec. Eng., Ferdowsi Univ. Mashhad, 2000.

[22] P. Kundur, Power System Stability and Control. New York: McGraw Hill, 1994.

[23] Anderson, P. M., and A. A. Fouad, Power System Control and Stability, Vol. 1. Ames, Iowa: The Iowa State University Press, 1977. 